\title{
EDGE EFFECT IN CHARGED-PARTICLE ANALYZING MAGNETS
}

\author{
C. M. BRAAMS \\ Fysisch Laboratorium der Rijks Universiteit, Utrecht, Netherlands \\ and
}

FOM-Instituut voor Plasma-Fysica, Rijnhuizen, Jutphaas, Netherlands*

Received 5 July 1963

The manner in which local saturation of pole pieces with sharp edges affects the fall-off of the magnetic induction in the fringing-field region is discussed and measured. Local saturation appears to set in at a field strength well below that at which over-all saturation of the pole pieces becomes noticeable. The

\section{Introduction}

In most magnetic beam analyzers or guiding systems for charged particles, the beam traverses not only the main field between iron pole pieces, but also a fringing field. If the apparatus is used at variable magnetic field strength, saturation of the iron may affect the fringing field and, hence, the ion-optical properties of the instrument. Three causes of field distortion may be distinguished; referring to fig. 1 , where representative field lines are sketched, we have:

Region $A$. The fringing field is reversed, compared to the main field; it vanishes if the yoke is of infinite permeability and increases, relative to the main field, if the yoke becomes saturated.

Region B. The fringing field has the same direction as the main field; it is proportional to the latter and to the gap width if the pole pieces are of infinite permeability and increases slightly, relative to the main field, if the pole pieces become saturated.

Region $\mathrm{C}$. The fringing field is affected by local saturation near the sharp edge of the pole; it decreases, relative to the main field, if the latter is increased.

In many cases, the fringing fields in regions $A$ and $B$ can easily be screened off. In this paper we will be concerned with the effect of local saturation

* Present address. resulting change in the calibration of a uniform-field sector magnet is discussed. A pole shape is proposed in which the magnetization is uniform and it is shown experimentally that no edge effect occurs with such poles.

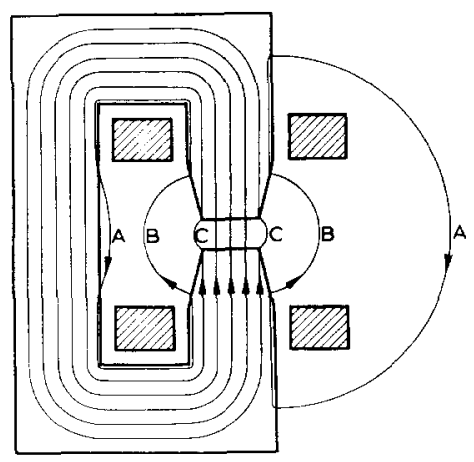

Fig. 1. Regions of different saturation effects; A. saturation of yoke, B. saturation throughout the pole picces, C. local saturation in pole edges.

in region $\mathrm{C}$, which occurs already at field settings below those at which saturation of the main portions of the iron becomes noticeable. We call this the edge effect. We will present experimental evidence for this phenomenon and discuss its importance for the calibration of a uniform-field analyzing magnet. We will also describe pole pieces designed to avoid non-uniform saturation and will show experimentally that no edge effect occurs in these poles.

\section{Design of Uniformly Saturating Poles Piece}

It is well-known that the magnetic field lines 
between iron pole faces tend to converge towards sharp edges, giving rise to maxima in the field strength limited only by local saturation of the iron. The depth of the saturated zone will increase with the excitation of the magnet, so that the field near the edge will vary less than proportional to the field deeper inside the gap.

The edge effect has been discussed extensively in connection with electrostatic problems. Rogowski ${ }^{1}$ ) has proposed a spark gap for breakdown studies, shaped so as to avoid maxima in the field strength at the edges of the electrodes. His conductors are derived from two infinite surfaces, parallel to the $z$-axis and intersecting the $x, y$-plane in

$$
x=\frac{a}{\pi} \varphi ; \quad y= \pm \frac{a}{\pi}\left(\frac{1}{2} \pi+\exp \varphi\right) .
$$

Here, $x, y$, and $z$ are cartesian coordinates, while $a$ is the minimum distance between the electrodes. The field pattern between these equipotential surfaces is described by

$$
x=\frac{a}{\pi}\left(\varphi+\mathrm{e}^{\varphi} \cos \psi\right), \quad y=\frac{a}{\pi}\left(\psi+\mathrm{e}^{\varphi} \sin \psi\right)
$$

from which equipotentials and field lines are found by putting $\psi=$ constant or $\varphi=$ constant. It can be shown that the flux is proportional to $\varphi$ and, hence, also to $x$ on the electrode surface. Therefore,

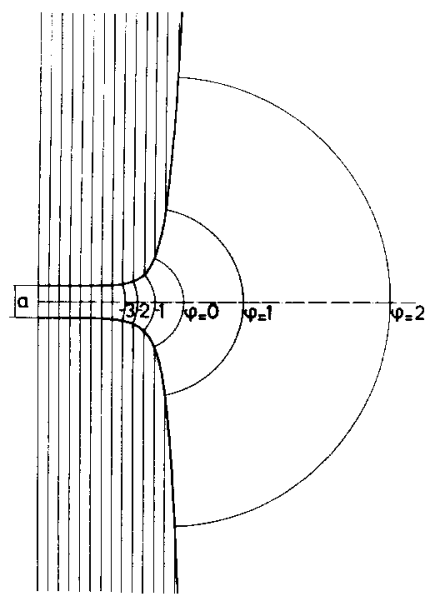

Fig. 2. Field of Rogowski poles, indicating uniform magnetization of the pole pieces.

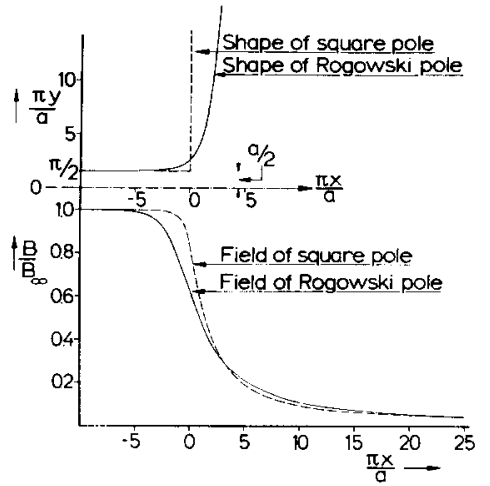

Fig. 3. Fringing field of Rogowski pole, compared with that of square pole.

Rogowski's formula, when applied to a magnet of infinite permeability, yields uniform magnetization of the iron throughout the pole piece.

The field lines of two such poles are shown in fig. 2 which illustrates the uniform field inside the iron. The field strength in the mid-plane between the pole faces is shown in fig. 3 , together with that produced by non-saturated square pole faces ${ }^{2}$ ). The comparison shows that the latter give a much steeper fall-off and a uniform-field region extending farther out towards the edge, for which they will often be preferred. However, in cases where changes in the fall-off at higher field settings cannot be tolerated, eq. (1) may serve as a guide towards the design of the magnet.

The minimum radius of curvature derived from eq. (1) equals $3 \sqrt{ } 3 a / 2 \pi$ or $0.83 a$. Hence, a simple prescription like tapering the sides of the pole pieces to a slope of $10 \%$ and rounding off the edges with a radius equal to the gap width will guarantee the absence of local saturation. This has been applied some years ago to the design of a beam analyzer $^{3}$ ) for a Van de Graaff ion accelerator. If a steeper fall-off is desired, or if the field strength is not pushed to the point of saturation of the body of the iron, one will make a compromise at a smaller radius, $r$. It may be estimated that the field setting,

1) W. Rogowski, Arch. f. Elektrotechnik, 12 (1923) 1.

2) Rothe, Ollendorff and Pohlhausen, Theory of tunctions (Technology Press, Massachusetts Inst. of Technology, 1948).

3) $\mathrm{H}$. van Rinsvelt and $\mathrm{Ph}$. B. Smith, paper submitted to Fysica, to appear in 1963. 
$B_{1}$ (measured in the uniform field), at which the edge will start to saturate, is related to the "saturation field strength", $B_{0}$, by

$$
B_{1}=r B_{0} / 0.83 a \text {. }
$$

\section{Experiment}

In order to measure the field distortion resulting from this edge effect, and to test the validity of the remedy proposed, an experiment has been carried out with a magnet designed for this purpose. Two pole pieces were constructed out of truncated pyramids (rectangular bottom plane, $9 \times 18 \mathrm{~cm}^{2}$; inclination of side planes, $\beta=\arctan 0.1$; height, $20 \mathrm{~cm}$; top plane, $5 \times 14 \mathrm{~cm}^{2}$ ). One of the long edges of each top plane was rounded off according to eq. (1) which $a=1 \mathrm{~cm}$ (see fig. 4). The poles

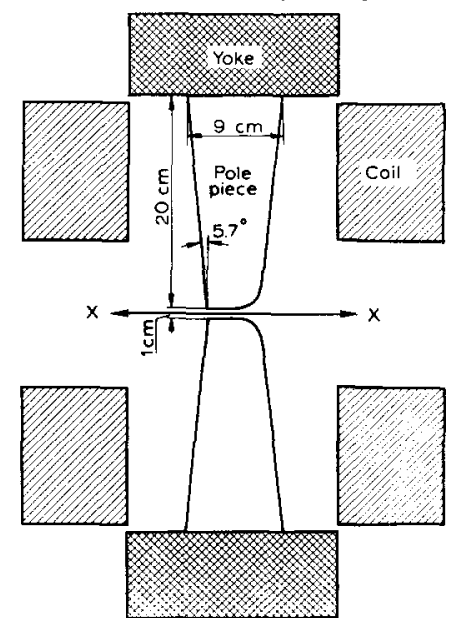

Fig. 4. Magnet used to compare fringing fields of sharp and rounded edges.

were made of low-carbon steel, which was annealed before the final machining was done. The yoke (two paths of $7.5 \times 20 \mathrm{~cm}^{2}$ cross section) was made of commercial soft steel. No tests of the magnetic properties of poles and yoke have been performed, but a comparison of measurements at increasing and decreasing fields has shown that the field shape was not noticeably affected by remanent magnetism.

With a moveable and a fixed search coil, both mounted on a shaft which could rotate at 40 cycles/ sec, the fringing field in the mid-plane, $B(x)$, was compared with the uniform field inside the gap, $B_{\infty}$. This was done by a compensation method, in which the ratio $B(x) / B_{\infty}$ was read on a helical potentiometer. At a given position, $x$, of the moveable coil, $B(x) / B_{\infty}$ was measured for different values of $B_{\infty}$, without $x$ being changed. Thus, the measurements did not rely on the reproducibility of $x$, so that deviations of one part in thousand in

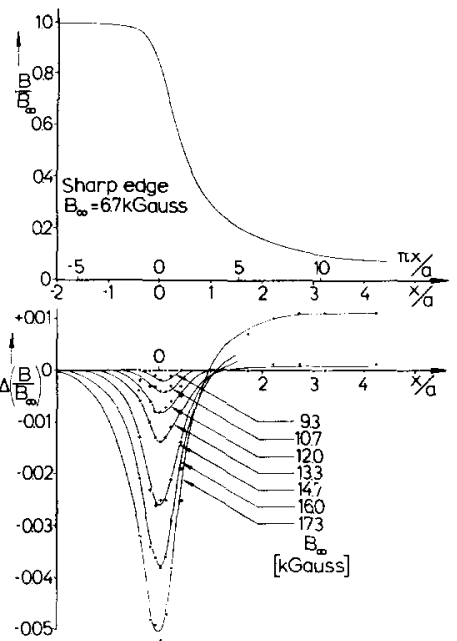

Fig. 5. Shape of fringing field (upper curve) and deviations resulting from saturation (lower set of curves) for sharp pole picces.
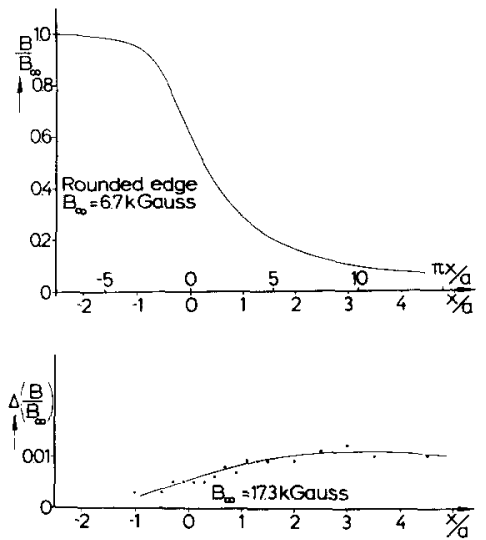

Fig. 6. Shape of fringing field (upper curve) and deviation resulting from saturation (lower curve) for Rogowski poles. 
the value of $B(x) / B_{\infty}$ could be detected. The magnetic field was varied in steps of $1.33 \mathrm{kG}$, and no change in the field shape was found for values of $B_{\infty}$ up to $8.0 \mathrm{kG}$. Hence the field shape at $6.7 \mathrm{kG}$ was considered to be undisturbed by saturation effects and was taken as the reference for measurements at higher field settings.

The undisturbed field has been plotted in the upper parts of figs. 5 and 6 for the sharp edge and the rounded edge, respectively. No attempt was made to measure the position of the coils with respect to the pole pieces, but changes in $x$ were measured on a vernier scale to $0.1 \mathrm{~mm}$. The zero of $x$ was determined, for the sharp edge by locating the position of maximum edge effect, and for the rounded edge by fitting the measured points of fig. 6 to the theoretical curve of fig. 3, which proved to be possible to within 1 percent in the range $-5<\pi x / a<10$.

Changes in the field shape as compared with that at $B_{\infty}=6.7 \mathrm{kG}$ have been plotted in the lower part of fig. 5 for different values of $B_{\infty}$. There first appears a dip at $9.3 \mathrm{kG}$, which increases in width and depth with increasing main-field setting. Up to $12.0 \mathrm{kG}$, however, the effect is restricted to the vicinity of the edge. Starting at about $13.3 \mathrm{kG}$, the far fringing field increases; at $17.3 \mathrm{kG}$, the deviation amounts to one percent of $B_{\infty}$ in a broad region extending far beyond $x / a=4$. This is interpreted as a result of saturation of the entire pole piece, as predicted for region $B$ of fig. 1 . The same increase is found in the field of the rounded edge. The first indication of it was noted at $13.3 \mathrm{kG}$, three out of seventeen measured points showing a positive deviation of 0.001 as compared to the field at $6.7 \mathrm{kG}$. Results of measurements at $17.3 \mathrm{kG}$ are plotted in the lower part of fig. 6 . Since no edge

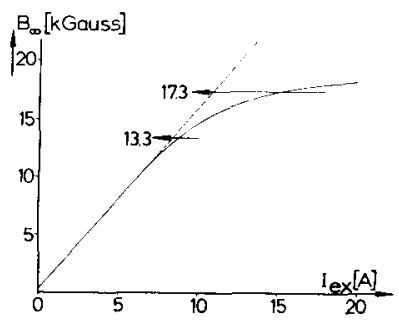

Fig. 7. Magnetization curve for magnet assembly. effect was evident from a comparison of data at $6.7,13.3$, and $17.3 \mathrm{kG}$, the measurements in the field of the rounded edge were not extended to other values of $B_{\infty}$. In order to give an impression of the accuracy, some of the measured points have been indicated in figs. 5 and 6 .

The saturation of the pole pieces above $13.3 \mathrm{kG}$ also shows up in fig. 7 in which $B_{\infty}$ is plotted against the excitation current (only the branch for decreasing $B_{\infty}$ has been drawn). From the date presented in fig. 5, the "displacement of the field boundary", defined here as

$$
h=\int_{x=-2 a}^{x=a} \Delta\left(B / B_{\infty}\right) \mathrm{d} x
$$

was computed. The limits of the integration were chosen so, that most of the edge effect but little of the effect of total pole saturation would be included.

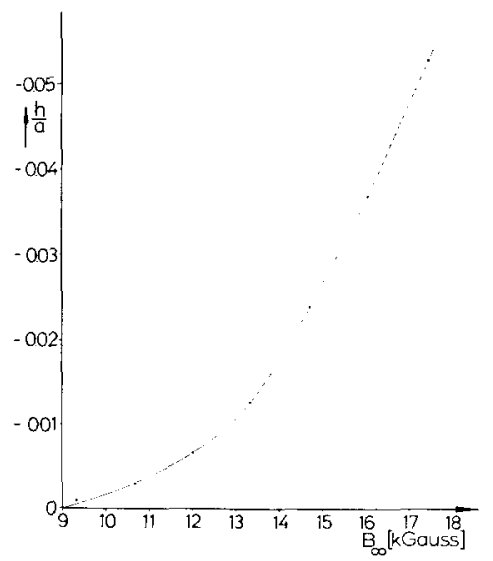

Fig. 8. Displacement of effective field boundary vs. main field setting.

Up to $13 \mathrm{kG}$ this definition provides no difficulties; at higher field settings, however, the particle trajectories are affected by changes in the field in regions $B$ and $C$ in a manner which depends on the location of the source and the detector and on the extend to which the stray fields are screened off. The meaning of $h$ then becomes questionable. In fig. $8, h / a$ has been plotted versus $B_{\infty}$. It should be noted that $h / a$ will certainly depend on the tapering angle, and in particular, that it will be appreciably greater for square pole pieces. One should expect 
also that the magnetization curve of the iron will affect the detailed shape of the curve shown in fig. 8. For this reason, we have not proceeded to study the edge effect for square or other pole shapes, having proved that it is entirely absent with the Rogowski poles. It would have been interesting, however, to investigate the result of rounding the edge with a smaller radius, or of shimming the pole pieces to extend the uniform-field region, which, of course, is not incompatible with rounding the edge.

\section{Edge Effect in a Beam Analyzer}

We consider a beam of charged particles, passing through entrance, and exit slits located on either side of a uniform-field sector magnet. We assume that the gap width is small compared to the trajectory between the slits, so that the field boundaries may be considered as infinitely sharp for the purpose of calculating the ion-optical properties of the analyzer. This subject has been discussed in detail for magnets with fixed field boundaries ${ }^{4}$ ). Following a method which was described more extensively elsewhere ${ }^{5}$ ) and of which we will here summarize what is relevant to the present problem, we will include the effect of a small displacement of the field boundaries in the firstorder theory.

A central trajectory is defined, which consists of a straight section of length $l^{\prime}$ between the entrance slit and the point of entrance in the field, a circular section of radius $r$ and deflection angle $\varphi$ in the uniform field, and another straight section of length $l^{\prime \prime}$ between the point of exit from the field and the exit slit. The normals to the field boundaries in the points of entrance and exit make angles $\varepsilon^{\prime}$ and $\varepsilon^{\prime \prime}$ with the straight sections of the central trajectory (see fig. 9). Restricting ourselves to the orbits in the central plane between the pole faces (in which plane also the central trajectory lies), we define the following first-order quantities:

$d^{\prime}$ and $d^{\prime \prime}$ : distances of an arbitrary trajectory to the slits;

$\alpha^{\prime}$ and $\alpha^{\prime \prime}$ : angles betwecn the straight sections of arbitrary, and central trajectory;

4) K. '. Bainbridge, part V of E. Segre, Experimental Nuclear Physics, Vol. 1 (1952) p. 559.

5) C. M. Braams, Thesis, Utrecht (1956).

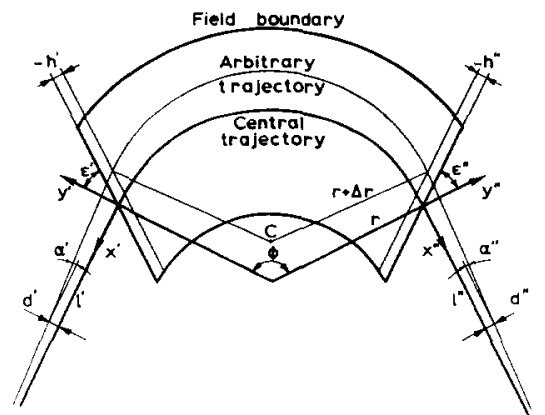

Fig. 9. Coordinate systems used to describe ion optics of uniform-field sector analyzer.

$\Delta r$ : difference between radii of arbitrary and central trajectory;

$h^{\prime}$ and $h^{\prime \prime}$ : displacements of the field boundaries. We assume

$$
\left\{\alpha^{\prime}, \alpha^{\prime \prime}\right\} \ll 1 \text { and }\left\{d^{\prime}, d^{\prime \prime}, \Delta r, h^{\prime}, h^{\prime \prime}\right\} \ll r .
$$

With respect to the signs, we refer to fig. 9, where all except $h^{\prime}$ and $h^{\prime \prime}$ are positive. Attaching $x^{\prime}, y^{\prime}$ and $x^{\prime \prime}, y^{\prime \prime}$ coordinate systems to the central trajectory, as indicated in fig. 9, we can derive relations between the first-order quantities by the following procedure. The coordinates $x_{\mathrm{c}}^{\prime}$ and $y_{\mathrm{c}}^{\prime}$ of the centre of curvature of the orbit are expressed in $d^{\prime}, \alpha^{\prime}, \Delta r$, and $h^{\prime}$; likewise, $x_{\mathrm{c}}^{\prime \prime}$ and $y_{\mathrm{c}}^{\prime \prime}$, in $d^{\prime \prime}, \alpha^{\prime \prime}, \Delta r$, and $h^{\prime \prime}$. Since these two sets of coordinates represent the same point, they must satisfy the transformation equations:

$$
\left(\begin{array}{c}
-x_{\mathrm{c}}^{\prime \prime} \\
y_{\mathrm{c}}^{\prime \prime}+r
\end{array}\right)=\left\|\begin{array}{rr}
-\cos \varphi & \sin \varphi \\
\sin \varphi & \cos \varphi
\end{array}\right\|\left(\begin{array}{c}
-x_{\mathrm{c}}^{\prime} \\
y_{\mathrm{c}}^{\prime \prime}+r
\end{array}\right) .
$$

Now, to first order, we have (omitting primes or double primes):

$$
\left(\begin{array}{c}
-x_{\mathrm{c}} \\
y_{\mathrm{c}}+r
\end{array}\right)=\left(\begin{array}{c}
d \tan \varepsilon+\alpha(l \tan \varepsilon+r)-h / \cos \varepsilon \\
d+\alpha l-r
\end{array}\right) .
$$

We note that $\varepsilon^{\prime}$ and $\varepsilon^{\prime \prime}$ are not necessarily small; we assume, however, that they are not much in excess of $\frac{1}{2} \pi$.

The change in calibration, $\Delta r$, caused by the edge effect $\left(h^{\prime}\right.$ and $\left.h^{\prime \prime} \neq 0\right)$ follows if one considers a beam entering through the entrance slit $\left(d^{\prime}=0\right)$ 
laong the central path $\left(\alpha^{\prime}=0\right)$ and calculates $\Delta r$ on the assumption that the beam passes again through the exit slit $\left(d^{\prime \prime}=0\right)$. The outgoing beam may differ in direction from the central path $\left(\alpha^{\prime \prime} \neq 0\right)$; one obtains two equations in $\alpha^{\prime \prime}$ and $\Delta r$, from which either one may be eliminated. Putting $\alpha^{\prime}=d^{\prime}=d^{\prime \prime}=0$ in (6), then substituting into (5) and solving for $\Delta r$, we find:

$\Delta r=\frac{\frac{l^{\prime \prime} h^{\prime \prime}}{\cos \varepsilon_{t^{\prime}}}+\left\{l^{\prime \prime} \cos \varphi+\left(l^{\prime \prime} \tan \varepsilon^{\prime \prime}+r\right) \sin \varphi\right\} \frac{h^{\prime}}{\cos \varepsilon^{\prime}}}{l^{\prime \prime} \tan \varepsilon^{\prime \prime}+r+l^{\prime \prime} \sin \varphi-\left(l^{\prime \prime} \tan \varepsilon^{\prime \prime}+r\right) \cos \varphi}$

Three special cases, which can be verified by simple trigonometry, may be noted. For a 90degree deflector $\left(\varphi=90^{\circ}\right)$, eq. 7 reduces to

$$
\Delta r=\frac{l^{\prime \prime} h^{\prime \prime} / \cos \varepsilon^{\prime \prime}+\left(l^{\prime \prime} \tan \varepsilon^{\prime \prime}+r\right) h^{\prime} / \cos \varepsilon^{\prime}}{l^{\prime \prime}+l^{\prime \prime} \tan \varepsilon^{\prime \prime}+r} .
$$

For a symmetric deflector $\left(h^{\prime \prime}=h^{\prime}=h ; \varepsilon^{\prime \prime}=\varepsilon^{\prime}\right.$ $=\varepsilon)$ :

$$
\Delta r=\cot \frac{1}{2} \varphi h / \cos \varepsilon .
$$

For a circular field boundary of radius $R$ and normal incidence, $\varepsilon=0$ and $\cot \frac{1}{2} \varphi=r / R$ so that

$$
\Delta r / r=h / R .
$$

We single out the latter case for an illustration of the importance of the edge effect. In the analyzer mentioned before ${ }^{3}$ ), the gap width, $a$, equals $1.5 \mathrm{~cm}$ and the radius of the circular field limit, $R, 33 \mathrm{~cm}$. As we have seen in the preceding section, $h$ may have negative values amounting to a few percent of $a$, whence $\Delta r / r$ becomes of the order of $-1 / 1000$. If the analyzer is calibrated at a low field setting, e.g., by means of known $Q$ values or reaction thresholds, and the momenta of particles selected at high field settings are calculated on the assumption that $r$ has not changed, the result may be high by 1 part in 1000 , or the energy by 2 parts in 1000 . This would lead to a systematic error in $Q$ value measurements which, at the present level of accuracy of such measurements, would be large enough to be of concern, although small enough to be easily overlooked, the more so because it would be overcome by total pole saturation at still higher field settings. Total pole saturation makes $h$ and $\Delta r$ positive; the measured energies then need an upward correction. On the other hand, saturation of the yoke (region C) again tends to make $\Delta r$ negative Finally, if the shape of the magnet differs from that of fig. 4 to the extend that the width of the pole faces in much greater than their height, saturation may effect the uniformity of the main field. The field strength in the region far from the yoke (or for a symmetric yoke, in the middle of the region of "uniform" field) may then be expected to show a depression and the effect of this on the calibration will depend on the position of the flux meter.

Measurements of reaction energies with the beam analyzer of the Utrecht Van de Graaff accelerator $^{3,6}$ ) show an indication of a negative $\Delta r$ at a field setting of $16 \mathrm{kG}$, which is ascribed to saturation. The location of the flux meter (far from the yoke) suggests that deviations in the main gap, as well as total pole saturation (region B), would give an effect in the opposite direction. Since we feel that the edge effect (region C) is excluded by the rounded edge, we are left with saturation of the yoke (region A) as the most likely cause of the suggested change in the calibration. Detailed field measurements on this particular magnet would be needed to clear up this point. Meanwhile, it may be considered to be a success of the design of this magnet that so far no indication of systematic error has been detected within the linear range of the magnetization curve; as we have shown in sec. 3 , sharp edges would have led to noticeable deviations already at much lower field settings (probably around 10 to $12 \mathrm{kG}$ ).

\section{Conclusions}

For the particular shape (tapering angle of $5.7^{\circ}$ ) and material of the pole pieces investigated, the edge effect has been detected at field settings of $9.3 \mathrm{kG}$ and upwards. At $9.3 \mathrm{kG}$, the displacement of the effective field boundary is 0.1 percent of the gap width, which is the limit of accuracy of our measurements. At $13.3 \mathrm{kG}$, it has grown to 1.3 percent, but there the onset of saturation of the whole pole pieces becomes noticeable. The field near the rounded edge was unaffected by saturation up to $13.3 \mathrm{kG}$. Rounding the edges is, therefore,

6) F. J. M. Snulers and P. M. Endt, Physica 28 (1962) 1093. 
shown to be a means for extending the useful range of a high-precision momentum analyzer.

\section{Acknowledgements}

This work was carried out as part of the research program of the Stichting voor Fundamenteel Onderzoek der Materie (FOM) with financial support from the Netherland's Organization for
Pure Scientific Research (ZWO) and Euratom. It was initiated while the author was connected with the University of Utrecht: the work described in sec. 3 was carried out in the Institute for Plasma Physics, under the association Euratom-FOM. The measurements were performed by Mr. S. van der Meulen. The material for the pole pieces was kindly supplied by DEMKA Steelworks, Utrecht. 